\title{
PINHEIROS ANTIGOS PODEM ENTRAR EM DECLÍNIO DEVIDO AO EFEITO DE MUDANÇAS CLIMÁTICAS
}

\author{
ANCIENT BRAZILIAN PINES MAY ENTER INTO DECLINE DUE TO CLIMATE CHANGE \\ EFFECTS
}

\author{
Alvaro Boson de Castro-Faria' ${ }^{1}$, Fagner Goes da Conceição², Olmar Antônio Denardin Costa ${ }^{3}$, \\ Solon Jonas Longhi ${ }^{4}$
}

1, 2, 3, 4 Universidade Tecnológica Federal do Paraná, Dois Vizinhos, Paraná, Brasil-alvarob@utfpr.edu.br, fagnerconceicao@utfpr.edu.br,odenardin@gmail.com\&longhi.solon@gmail.com

\section{RESUMO}

A saúde florestal precisa ser considerada nas estratégias de conservação in situ da Araucaria angustifolia, e eventos climáticos extremos podem influenciar processos de estresse em árvores maduras, com baixo poder de recuperação. Apresenta-se um diagnóstico fitossanitário de pinheiros antigos em uma área de eminente valor ecológico em Palmas, PR, permitindo comparações futuras sobre a frequência dos pinheiros desvitalizados e mortos. Foi verificado o comportamento de variáveis meteorológicas, entre 2008 a 2018. Os dados foram organizados pela ANOVA e por testes de médias. Foi realizada uma Análise de Agrupamento, utilizando a distância euclidiana como medida de similaridade, e formados os grupos, foi realizado uma Análise de Componentes Principais, para ordenação das variáveis. As alterações aqui reportadas foram para os meses de inverno, onde no Sul do Brasil, as chuvas são provocadas principalmente pela interação entre as massas de ar polar, com as massas continentais condicionadas a eventos de $E I N$ Niño. As mudanças foram cíclicas e padrões puderam ser verificados. $O$ calor e a secura do ar precisam ser monitorados por mais tempo, para a constatação do aquecimento climático, porém, verificou-se que chuvas concentradas em períodos curtos podem ter influenciado a desvitalização e condicionado árvores à murchas por ressecamento, devido às falhas hidráulicas, o que aumentaria a mortalidade no longo prazo. Existem indícios de que os invernos de 2009 e de 2015 foram estressantes às araucárias maduras e de grande porte, dada à irregularidade na distribuição das chuvas e aos períodos de secas. Ainda, que uma próxima estiagem prolongada poderá ser verificada até o inverno de 2021.

PALAVRAS-CHAVE: Araucaria angustifolia, El Niño, Espécies ameaçadas, Estiagens, Proteção e saúde florestal.

\section{ABSTRACT}

Forest health needs to be considered in the in situ conservation strategies for Araucaria angustifolia, and climate changes anomalies may be influencing drought-induced tree mortality in the mature trees, with low recovery power. We present a phytosanitary assessment for a native forest in Palmas (State of Paraná), with ancient trees, for future comparisons over the dead ones. It was verified the behavior of meteorological variables between 2008 and 2018. The data series were organized by ANOVA and by average tests. A Grouping Analysis was performed, using the Euclidean distance as a similarity pattern, obtaining the groups. A Principal Component Analysis was performed to order the variables. The changes reported here were for the winter months, where in southern Brazil, rainfall is mainly caused by the interaction between the polar air masses, with the continental masses conditioned to ENSO events. The changes were cyclical and standards were verified. Air heat and dryness needs long-term monitoring for climatic warming statement, but, it was verified that overconcentrated rainfall in short periods may have influenced the devitalization and conditioned trees to wilt by dryness, due to hydraulic failure, which would increase mortality in the long term. There are indications that 2009 and 2015 winters were stressful to the Brazilian pine, due to the irregular distribution of the rains and the droughts periods. Still, that a next prolonged drought could be verified until the winter of 2021.

KEYWORDS: Araucaria angustifolia, EI Niño, Threatened species, Droughts, Forest health and protection. 


\section{INTRODUÇÃO}

A defesa do bem-estar florestal ainda não foi considerada nas análises ambientais e nas políticas públicas de conservação in situ de espécies da flora especialmente protegida e ameaçada de extinção. Dentro de uma perspectiva interdisciplinar, este trabalho buscou integrar as áreas de conhecimento de Proteção Florestal e Climatologia.

A conífera Araucaria angustifolia (Bertol.) Kuntze é uma das principais espécies da flora brasileira, visto seu valor cultural, ambiental e econômico. Historicamente, o aproveitamento desta árvore foi fundamental para o desenvolvimento da região Sul do Brasil. Tendo como referência uma agenda conservacionista que permita o uso sustentável das florestas, notadamente com compromissos internacionais assinados pelo governo federal, em consideração à Convenção sobre a Diversidade Biológica (CDB) (BRASIL, 1998), o Ministério do Meio Ambiente (MMA) baixou a Portaria n. 43/2014, instituindo o Programa Nacional de Conservação das Espécies Ameaçadas de Extinção. A lista da flora ameaçada, por sua vez, foi reconhecida em sua última versão pela Portaria MMA n. 443/2014, com a presença da espécie $A$. angustifolia, classificada como "Em Perigo (EN)".

Estudos sobre a vulnerabilidade de ecossistemas tem ganhado relevância pela comunidade científica, e grupos de pesquisa destacam a importância das análises interdisciplinares, com o objetivo de monitorar padrões e tendências da mortalidade de árvores induzida pelo clima (HARTMANN et al., 2015; AUBIN et al., 2018; HARTMANN et al., 2018a). O quinto Relatório de Avaliação do Painel Intergovernamental sobre Mudanças Climáticas (IPCC, 2014) considerou que é extremamente provável (entre 90 a $100 \%$ de probabilidade) que estas mudanças globais sejam decorrentes de causas antropogênicas.

A detecção do nível de intensificação dos estresses nas florestas nativas torna-se um desafio cada vez mais importante para a sociedade, uma vez que estas podem levar décadas para restaurarem os serviços ecossistêmicos que providenciavam (TRUMBORE et al., 2015). Segundo Fang et al. (2018), os riscos à saúde das florestas naturais estão cada vez mais intensos, favorecendo o declínio e a mortalidade destes ecossistemas em muitas regiões do planeta.

Declínios de desenvolvimento e mortalidade natural em araucárias geralmente ocorrem em árvores fisiologicamente maduras, sendo que o vigor vegetativo de recuperação é mais evidente na fase juvenil. Por isso, em florestas, as árvores impactadas são predominantemente das classes dominantes e codominantes, as maiores do sítio. Já as árvores jovens, demandam menos recursos para se recuperarem (CASTRO-FARIA, 2018). A Síndrome do declínio foi descrita por Manion (1991) como a interação de um número intangível de fatores abióticos e bióticos que causam a deterioração e a morte das árvores. Os fatores que levam ao estresse crônico das árvores podem resultar em sinais e sintomas parecidos, quer seja a debilidade e diminuição de crescimento, diebacks e murchas por ressecamento. A maioria dos declínios está associada a algum tipo local ou regional de anomalia relacionada a fatores ambientais externos, que causam disfunção fisiológica em partes específicas das plantas.

Partindo-se da hipótese de que o aumento de temperatura atmosférica no planeta poderia prejudicar o desenvolvimento das araucárias, Fritzsons \& Wrege (2017) ratificaram que as regiões onde os pinheiros são mais encontrados naturalmente, estão sempre associadas para com altitudes a partir de $500 \mathrm{~m}$ e com temperaturas baixas ou amenas, evidenciando a preferência da espécie em desenvolver-se em locais frios. Em outro estudo, Fritzons et al. (2018) subdividiram o Paraná em quatro regiões climáticas de ocorrência da espécie, destacando que regiões onde a oferta hídrica no inverno seja maior, favorecerá a adaptabilidade das árvores. Em outra análise, Wrege et al. (2017) estimaram que no longo prazo o clima no Paraná se tornará mais quente e seco, e muitas áreas de ocorrência da Floresta Ombrófila Mista poderão se converter em Floresta Estacional Semidecidual.

Dale et al. (2001) e Sugden et al. (2015) atribuem a ocorrência de distúrbios biológicos como efeitos diretos das mudanças climáticas, causadas por estiagens decorrentes do aquecimento global, ao nível local. Brando et al. (2014) detectaram os efeitos das queimadas sobre os regimes de estiagens na região amazônica e na mortalidade de árvores. Maia et al. (2018) lembraram os danos ambientais por conta das secas prolongadas no estado de São Paulo, tendo destacado que a resiliência e adaptação das culturas agrícolas às mudanças do clima dependerá da manutenção e garantia dos serviços ecossistêmicos.

Salviano et al. (2016) defendem que o clima no Brasil está ficando mais quente e seco, mas que as chuvas na região Sul poderão ficar mais intensas. Dados de Fritzsons et al. (2011) indicam que a região Norte do Paraná é a mais suscetível à períodos de estiagens. Chuvas sazonais são previstas no litoral e na região Oeste. No Centro do 
estado, existe uma zona de transição, onde estiagens podem ser influenciadas por inúmeros fatores.

No tocante à alteração nos regimes de secas e para a região Sul brasileira, duas hipóteses precisariam ser confirmadas, para se crer nos efeitos das mudanças climáticas sobre o declínio em longo prazo das populações do pinheiro brasileiro. A primeira seria a de que a falta de água estaria influenciando como causadora de estresse, em árvores maduras, com baixo poder de recuperação e resiliência. A segunda hipótese é a de que o aquecimento global já esteja expressando significativamente alterações na disponibilidade hídrica ao nível local e regional (CASTRO-FARIA, 2018).

Esta pesquisa apresenta um diagnóstico ambiental do estado fitossanitário de araucárias antigas e de grande porte em uma área de eminente valor ecológico localizada em Palmas, PR, permitindo comparações futuras dos efeitos climáticos sobre a frequência das desvitalizadas e das mortas. Como objetivo específico, este trabalho buscou verificar o comportamento climático das variáveis relacionadas ao aumento da temperatura, e à diminuição da umidade e da precipitação pluviométrica, para a região de estudo, entre os anos de 2008 a 2018, e apresentar argumentos para a análise do aquecimento global e de mudanças climáticas sobre a vulnerabilidade das árvores maduras.

\section{MATERIAL E MÉTODOS}

\section{Área de estudo e diagnóstico de árvores maduras}

O estudo foi realizado em um remanescente de Floresta Ombrófila Mista (FOM) secundária em estágio avançado de sucessão, com altitude entre 957 a 1094 m, localizado em uma grande propriedade rural no município de Palmas, na divisa entre as mesorregiões centro sul e sudeste do Paraná, inserida na bacia dos afluentes do médio rio Iguaçu (Figura 1 ). O clima na região é do tipo $\mathrm{Cfb}$ segundo a classificação de Köppen (ALVARES et al., 2013), com geadas frequentes no inverno.

O imóvel pertence às Indústrias Pedro Pizzatto Ltda, com uma extensão de 716,3 hectares, tendo divisa ao norte com o município de Bituruna, e ao leste, com General Carneiro. Esta área é conhecida por conservar uma floresta natural com araucárias centenárias, já tendo sido objeto de pesquisas de longa duração (DALLA-CORTE et al., 2014). Para Barth-Filho (2002) a área basal deste fragmento de mata atlântica em 1998 possuía 25,71 $\mathrm{m}^{2} /$ ha, sendo que destes, $13 \mathrm{~m}^{2} /$ ha eram pinheiros (52\%), e a altura média destas arvores eram de 15,73 m. Para
Mognon et al. (2013), as araucárias representam cerca de $65 \%$ dos estoques de carbono daquele remanescente.

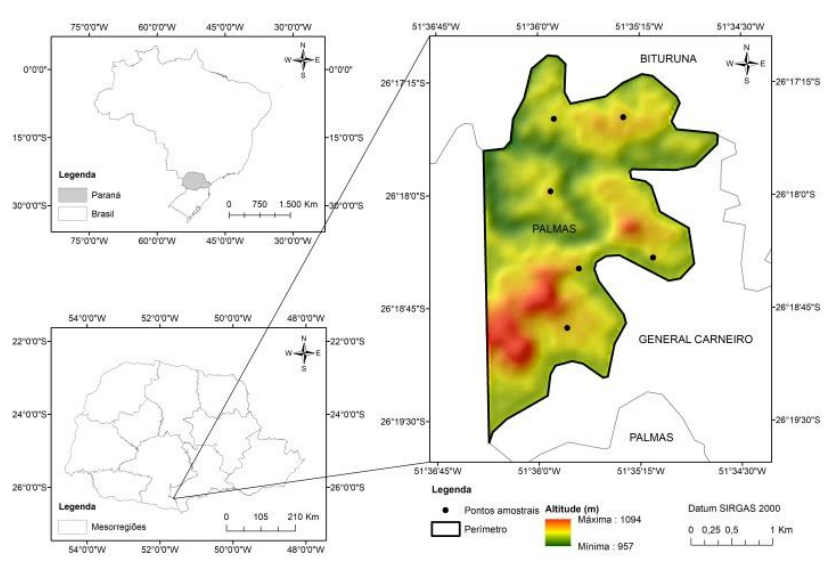

Figura 1. Caracterização da área de estudo.

Foram distribuídas aleatoriamente e em única ocasião, seis parcelas de $1.000 \mathrm{~m}^{2}(50 \times 20 \mathrm{~m})$, onde foram avaliadas as araucárias maduras, ou seja, com crescimento já estabilizado. Estes dados foram coletados em setembro de 2017 (final do inverno). Mensuraram-se por indivíduo, o diâmetro à altura do peito, a altura total e o estado fitossanitário, onde as árvores foram classificadas como: saudáveis, estressadas, desvitalizadas ou mortas, conforme o protocolo para o diagnóstico da saúde de pinheiros maduros apresentado por Castro-Faria (2018), descrito a seguir.

- Árvore Saudável: não apresenta sinais de injúrias ou sintomas de ataques de agentes bióticos; os ramos e folhas estão vigorosos;

- Árvore Estressada: apresenta sinais de injúrias ou sintomas de ataques de agentes bióticos; porém, a árvore pode se recuperar naturalmente e irá ainda permanecer viva por tempo indeterminado;

- Árvore Desvitalizada: apresenta sinais de injúrias ou sintomas de ataques de agentes bióticos; Não se pode definir se a árvore conseguirá se recuperar naturalmente, árvore apresenta seiva e certo grau de atividade fisiológica;

- Árvore Morta: a raiz está comprometida definitivamente; independente de possuir seiva ou estiver seca, a árvore não consegue mais assimilar os recursos necessários para se recuperar de injúrias físicas ou biológicas, associadas à desvitalização.

\section{Análises climatológicas}

Danos ambientais e declínios em florestas foram reportados, associados ao aquecimento global e em 
regiões frias e de latitudes acima de $23,5^{\circ}$ referente às regiões acima do trópico de Câncer e abaixo do de Capricórnio (HARTMANN et al., 2015; GENTILESCA et al., 2017; BELL et al., 2018; NELSON et al., 2018). Para Westerling et al. (2006), existe o aumento de risco de incêndios florestais no início da primavera na região norte das montanhas rochosas americanas.

Na América Latina, Pérez \& Arnesto (2017) e Meza et al. (2018) analisaram os efeitos das alterações climáticas sobre os ecossistemas chilenos. Por estes argumentos e considerando a expectativa de que as mudanças nos padrões do clima seriam mais bem verificadas na estação fria, optou-se por trabalhar apenas com os dados climáticos de inverno (séries de julho, agosto e setembro), organizando-os em relação à média para cada dois dias (48 h). Este agrupamento permitiu a obtenção de séries anuais com 39 a 46 repetições a depender do parâmetro de interesse.

Foi utilizada a série de parâmetros climáticos da estação automática instalada em General Carneiro, cedidos pelo Instituto Nacional de Meteorologia (INMET), localizada cerca de $40 \mathrm{~km}$ da área de estudo. O registro histórico representou o interstício compreendido entre 2008 a 2018, estando organizado em séries horárias. Os parâmetros analisados foram: temperatura do ar mínima absoluta, a instantânea (para a hora cheia), a máxima absoluta, a umidade atmosférica mínima, e dados de precipitação (total do trimestre, média e máxima).

A normalidade dos dados foi testada com o auxílio do procedimento UNIVARIATE (SAS, 2013), e os dados transformados foram utilizados na estimação dos intervalos de confiança mínimos e máximos a 95\% de probabilidade, com série temporal organizada em diagramas de caixa. Para a análise de variância, utilizou-se o procedimento GLIMMIX (SAS, 2008). Foi utilizada a versão acadêmica do SAS. Gráficos de tendências foram elaborados no software Excel. Também foram indicados os valores absolutos extremos, sendo para estes, inseridas linhas de tendência polinomial de sexto grau.

Também foi utilizada análise multivariada, para tanto, foi elaborada uma matriz contendo onze linhas, constituídas pelos anos de 2008 a 2018, e seis colunas, constituídas pelas variáveis climáticas: Tmax-máxima, Tmédia-instantânea, Tmin-mínima, UR-mínima e Precipitação-máxima. Foi realizada Análise de Agrupamento pelo método hierárquico aglomerativo, utilizando a distância euclidiana como medida de similaridade e o método de ligação Ward para a obtenção de um dendrograma. Uma vez obtidos os grupos, foi realizada Análise de Componentes Principais pela técnica de Escala Ideal, para ordenação das variáveis climáticas analisadas em seus respectivos anos de avaliação (JOHNSON \& WICHERN, 2007; RIBAS \& VIEIRA, 2011).

\section{RESULTADOS E DISCUSSÃO}

Foi estimada a existência de 2,6 a 5,3 \% de árvores mortas por causas naturais na área estudada. Os resultados descritivos desta caracterização encontram-se na tabela 1.

Tabela 1. Caracterização da saúde das árvores maduras na área de estudo.

\begin{tabular}{ccccccc}
\hline \multicolumn{7}{c}{ Unidade Amostral } \\
\hline Classe & Média & Variância & Erro padrão & CV\% & $\begin{array}{c}\text { IC } \\
\text { mín }\end{array}$ & $\begin{array}{c}\text { IC } \\
\text { máx }\end{array}$ \\
\hline SD & 5,2 & 4,6 & 0,9 & 41,4 & 2,7 & 7,6 \\
ES & 0,5 & 1,5 & 0,6 & 245 & - & 1,9 \\
DV & 0,7 & 1,1 & 0,5 & 155 & - & 1,9 \\
MO & 0,2 & 0,2 & 0,2 & 245 & - & 0,6 \\
\hline \multicolumn{7}{c}{ Árvores por Hectare } \\
\hline Classe & Média & Máximo & \%Média & \%MP & DAP & AT \\
(cm) & (m) \\
\hline SD & 51,7 & 76,2 & 79,5 & 63,4 & 67 & 23 \\
\hline ES & 5,0 & 19,1 & 7,7 & 15,9 & 50 & 20 \\
\hline DV & 6,7 & 18,5 & 10,3 & 15,4 & 66 & 22 \\
MO & 1,7 & 6,4 & 2,6 & 5,3 & 51 & 18 \\
\hline Total & $\mathbf{6 5 , 0}$ & $\mathbf{1 2 0 , 2}$ & $\mathbf{1 0 0}$ & $\mathbf{1 0 0}$ & \\
\hline SD & & & & \\
\hline
\end{tabular}

$\mathrm{SD}$ = saudável; $\mathrm{ES}$ = estressada; $\mathrm{DV}$ = desvitalizada; $\mathrm{MO}=$ morta; $\mathrm{MP}=$ máxima ponderada; $\mathrm{DAP}=$ diâmetro médio à altura do peito; $A T$ = altura total média; e IC = intervalo de confiança $(\mathrm{GL}=5 ; 0,05)$.

A série indicou um padrão em que as temperaturas tenderam a ir aumentando em ciclos de quatro anos, ocorrendo uma queda na estação seguinte, indicando um novo período. Ao se analisar as temperaturas médias (Figura 2), este comportamento foi verificado entre os interstícios de 2008 a 2011, entre 2012 a 2015, e entre 2016 e 2017. Em termos gerais, os anos de 2008, 2012 e 2016 foram os mais frios ( $F=71,01, p<0,05)$, enquanto em 2014, houve o inverno mais quente, com $20,5{ }^{\circ} \mathrm{C}$ (Tabela 2). 


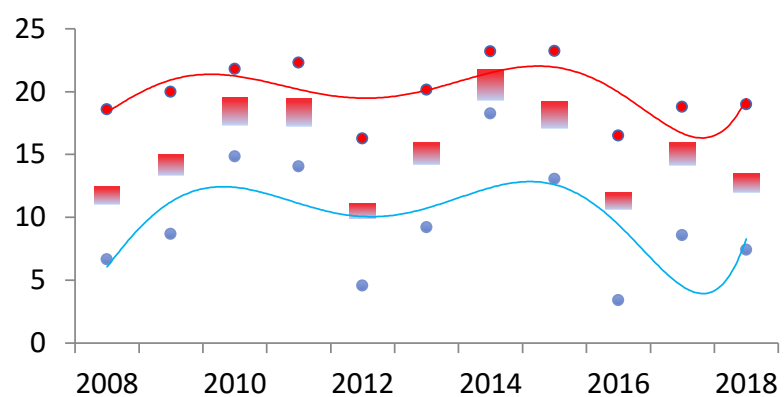

Figura 2. Temperatura do ar instantânea média $\left({ }^{\circ} \mathrm{C}\right)$. Em que: $\mathrm{BOX}=$ intervalo entre IC-min e IC-máx; Pontos = mínimos e máximos absolutos; e Polinômio = tendência entre os extremos de máxima (linha vermelha) e de mínima (linha azul).

Tabela 2. Parâmetros climáticos na estação de inverno e para o período de avaliação.

\begin{tabular}{|c|c|c|c|c|c|}
\hline Ano & \multicolumn{2}{|c|}{ T.méd $\left({ }^{\circ} \mathrm{C}\right)$} & T.máx $\left({ }^{\circ} \mathrm{C}\right)$ & T.min $\left({ }^{\circ} \mathrm{C}\right)$ & UR\% mín \\
\hline 2008 & \multicolumn{2}{|c|}{$12,1 \mathrm{a} b$} & $21,5 a b$ & $5,7 a b$ & $46,9 a b$ \\
\hline 2009 & 14,4 & $c d$ & 20,6 a & 6,6 a b c & $54,8 \quad b$ \\
\hline 2010 & 18,5 & e & $22,4 \mathrm{a} b$ & 7,0 a b c d & 46,6 a b \\
\hline 2011 & 18,5 & e & $22,3 \mathrm{a} \mathrm{b}$ & 7,3 bcd & $47,0 \mathrm{a} b$ \\
\hline 2012 & \multicolumn{2}{|c|}{$10,9 a$} & $24,0 \quad b$ & 7,1 bcd & $45,5 \mathrm{a} b$ \\
\hline 2013 & 15,3 & $d$ & 22,6 a b & $6,9 \mathrm{abcd}$ & 49,8 a b \\
\hline 2014 & 20,5 & $f$ & $23,4 \mathrm{ab}$ & $7,9 \mathrm{bcd}$ & $50,6 \mathrm{a} b$ \\
\hline 2015 & 18,3 & e & $24,2 \quad b$ & $9,4 \quad c d$ & $43,7 a$ \\
\hline 2016 & \multicolumn{2}{|c|}{11,7 a b } & 22,6 a b & $4,6 a$ & $42,9 a$ \\
\hline 2017 & 15,2 & $d$ & $22,9 \mathrm{a} b$ & $8,3 \quad c d$ & $48,9 a b$ \\
\hline 2018 & 13,1 & $\mathrm{bc}$ & $22,4 \mathrm{a} b$ & 5,8 a b c & 48,8 a b \\
\hline $\mathrm{F}_{\text {(calc.) }}$ & 71,01 & & $2,59^{*}$ & $5,67^{*}$ & $2,27^{*}$ \\
\hline CV (\%) & 16,6 & & 17,8 & 49,7 & 28,9 \\
\hline
\end{tabular}

Dados referentes a média para cada $48 \mathrm{~h}$. Médias com letras diferentes na coluna diferem significativamente pelo teste de Tukey a 95\% probabilidade.; ${ }^{*}=$ significativo $(p<0,05)$; ns = não significativo; e $\mathrm{CV}(\%)=$ coeficiente de variação.

Quanto aos períodos extremos, em 2015 a temperatura máxima indicou um ápice de $24,2{ }^{\circ} \mathrm{C}(\mathrm{F}=$ 2,59, $\mathrm{p}<0,05$; Figura 3). Em comparação às temperaturas mínimas, o ano seguinte de 2016 registrou $4,6^{\circ} \mathrm{C}$, tendo sido o ano mais frio da série $(F=5,67, p<0,05$; Figura 4). Vale registrar que apesar de 2012 ter apresentado o inverno mais frio, a temperatura máxima-média registrada foi de $24^{\circ} \mathrm{C}$, sendo equivalente à máxima do ano de 2015.

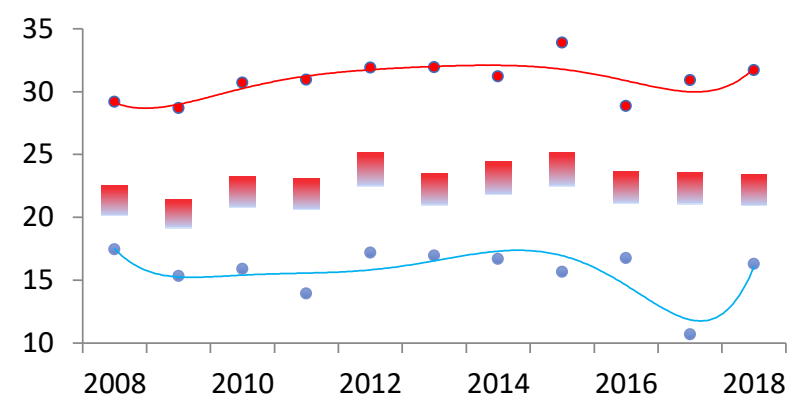

Figura 3. Temperatura do ar máxima absoluta $\left({ }^{\circ} \mathrm{C}\right)$. Em que: $\mathrm{BOX}=$ intervalo entre IC-min e IC-máx; Pontos = mínimos e máximos absolutos; e Polinômio = tendência entre os extremos de máxima (linha vermelha) e de mínima (linha azul).

Ainda quanto aos extremos de frio, houve indícios de que os primeiros oito anos da série apresentaram a tendência de aumento progressivo das temperaturas, entre 2008 a 2015 (Figura 4).

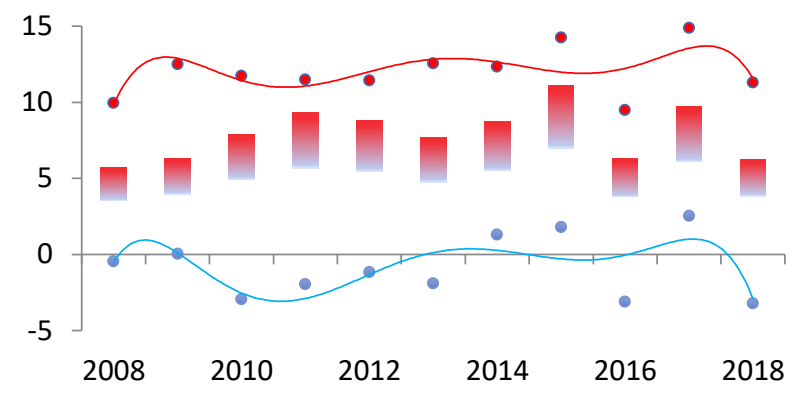

Figura 4. Temperatura do ar mínima absoluta $\left({ }^{\circ} \mathrm{C}\right)$.

Em que: $\mathrm{BOX}=$ intervalo entre IC-min e IC-máx; Pontos = mínimos e máximos absolutos; e Polinômio = tendência entre os extremos de máxima (linha vermelha) e de mínima (linha azul).

O comportamento da umidade relativa atmosférica daquela estação automática indicou que 2009 foi o ano mais úmido (54,8\%) e os anos de 2015 e 2016 foram os mais secos (43,7\% e 42,9\%; $F=2,27, p>0,05$; Figura 5).

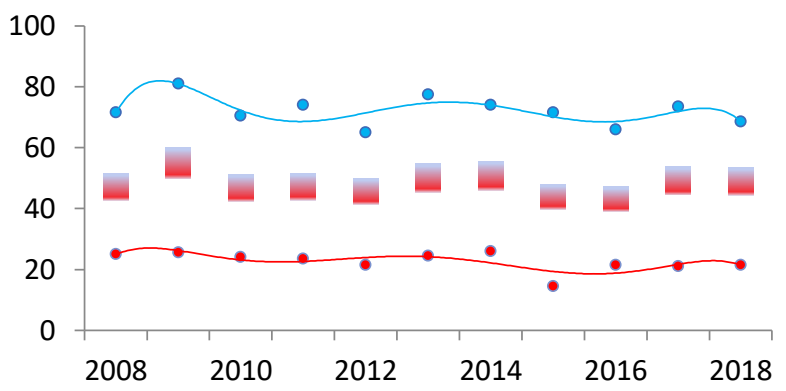

Figura 5. Umidade relativa atmosférica mínima (\%). Em que: BOX = intervalo entre IC-min e IC-máx; e Polinômio = tendência entre os extremos de máxima (linha azul) e de mínima (linha vermelha). 
A maior estiagem ocorreu em 2015 , com precipitação total inferior a $50 \mathrm{~mm}$. Chuvas entre 50 a $100 \mathrm{~mm}$ foram observadas nos anos de 2009, 2010 e 2017. Patamares de precipitação entre 100 a $150 \mathrm{~mm}$ ocorreram nos anos de 2012 e 2018 . Entre 150 a $200 \mathrm{~mm}$ foram verificados em 2008 e 2011. Os períodos mais chuvosos foram em 2013, 2014 a 2016 (Figura 6). A tendência considerando as máximas absolutas a cada $48 \mathrm{~h}$ indicou que para o longo prazo, existiu um comportamento cíclico variável a cada seis anos, na intensidade de distribuição pluviométrica.

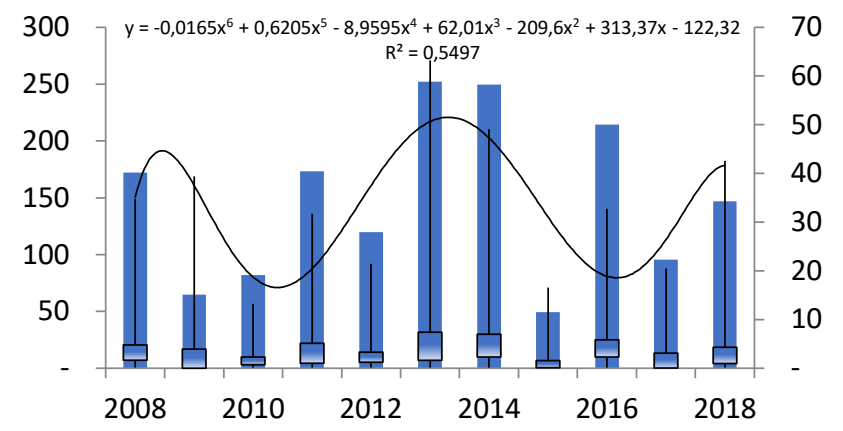

Figura 6. Precipitação pluviométrica (no inverno).

Em que: Coluna = precipitação total da estação $(\mathrm{mm}) ; \mathrm{BOX}=$ Intervalo entre IC-Mínimo e IC-máximo; Eixo vertical = máximo a cada 48 h; e Polinômio = tendência de chuvas máximas.

A análise de agrupamento classificou a série em três grupos distintos entre si, sendo o primeiro referente aos anos de 2010/12/15/17, caracterizado com picos de precipitação inferior a $20 \mathrm{~mm}$ para cada $48 \mathrm{~h}$. O Grupo 2 se referiu aos anos de 2008/09/11/16/18, sendo estes com chuvas máximas entre 20 a $50 \mathrm{~mm}$. E, o Grupo 3, aos anos de 2013 e 2014, mais chuvosos, com chuvas superiores a $50 \mathrm{~mm} / 48 \mathrm{~h}$ (Figura 7).

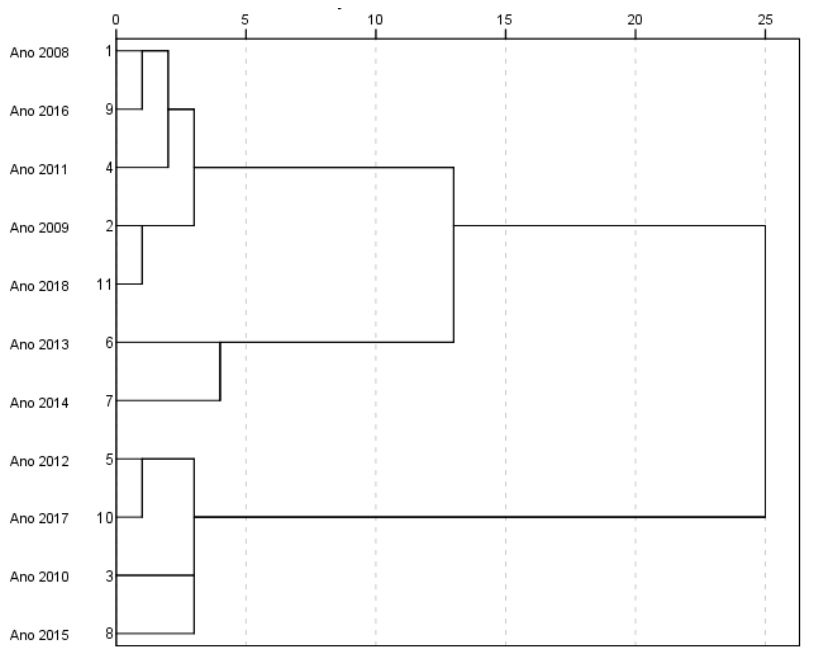

Figura 7. Dendrograma com ligação de Ward: combinação de cluster de distância redimensionada.

Em que: Grupo 1 (chuvas < 20 mm: 2010/12/15/17); Grupo 2 (entre 20 a 50 mm: 2008/09/11/16/18); Grupo 3 (chuvas concentradas > $50 \mathrm{~mm}: 2013 / 14$ ).
A Figura 8 indica que os anos de 2010 (3) e 2015 (8), à direita no gráfico, estiveram influenciados principalmente pela temperatura máxima-máxima, isto é, foram anos quentes (Figura 3) e pouco chuvosos (Figura 6). 0 ano de 2017 (10) foi influenciado pela temperatura mínimamínima, o de 2014 (7), pela temperatura média, e 2009 (2), pela UR-mínima (estiagem). No entanto, a precipitação máxima a cada $48 \mathrm{~h}$ (pancadas de chuva), foi o parâmetro que mais influenciou o clima na maior parte do período verificado.

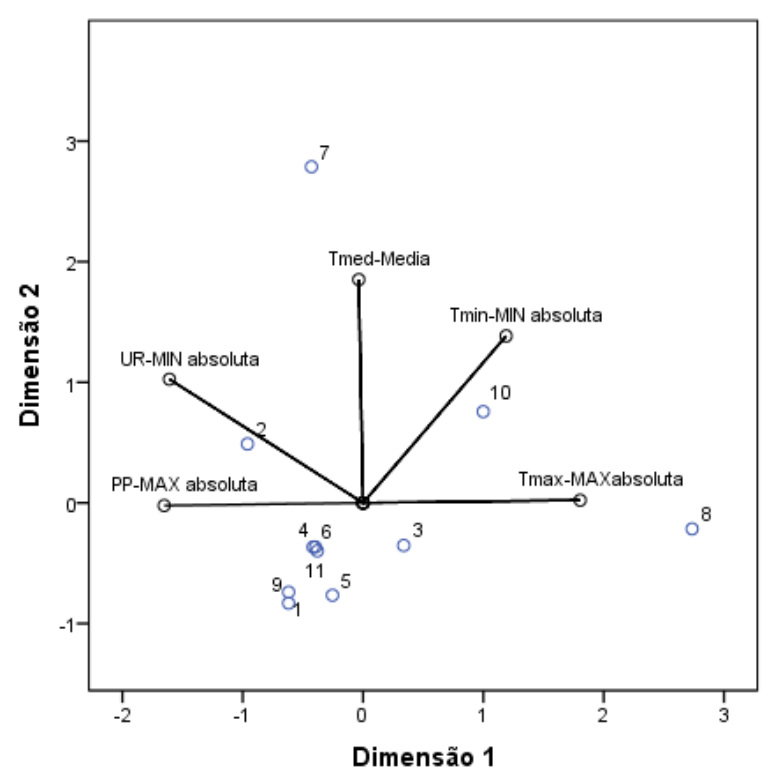

Figura 8. Análise biplot de carregamento dos componentes principais.

Em que: $1=2008,2=2009,3=2010,4=2011,5=2012,6=$ $2013,7=2014,8=2015,9=2016,10=2017,11=2018$.

Os dados climáticos representam um período muito curto de avaliações e não permitem afirmar se de fato está ocorrendo o aumento da temperatura e a diminuição da umidade relativa na região, por efeitos do aquecimento global. Para uma melhor constatação, tornase imprescindível o acompanhamento contínuo e de longo prazo, destes parâmetros meteorológicos.

O comportamento das temperaturas culminou em ondas de calor verificadas no biênio 2014/15, sendo os anos mais quentes da série. Apesar de ser menos perceptível no parâmetro da umidade, o período mais seco se deu na sequência bianual, compreendida entre $2015 / 16$. Ao se analisar a evolução do comportamento das chuvas, a tendência indicou os anos de estiagens de inverno (total < $100 \mathrm{~mm}$ ), verificados em 2009/10/17, e mais gravemente, em 2015 (total < $50 \mathrm{~mm}$ ).

Ao tentar explicar o padrão climático na região de estudo, acredita-se que este esteja sendo influenciado 
pelo fenômeno conhecido como El Niño de Oscilação Sul (ENOS), evento cíclico decorrente do aquecimento do oceano Pacífico e que influencia na ocorrência de chuvas em territórios continentais tropicais. Shenkin et al. (2018) afirmam que devido ao aquecimento global, existe uma expectativa de verificação de temperaturas mais quentes e mais períodos severos de estiagens nestas regiões, resultando em oscilações climáticas na zona de convergência intertropical do hemisfério Sul. Os autores analisaram a interação entre clima e regime de manejo em 2004/5, na floresta amazônica boliviana, indicando que a altura das árvores favoreceu o seu estresse às secas, principalmente em áreas não submetidas às intervenções (desbastes). Para Malthi et al. (2018), o estresse causado por secas e incêndios sobre as florestas tropicais da região leste amazônica, em uma escala global, podendo interferir no ciclo hidrológico e de captura do carbono. Eventos severos de El Niño também ocorreram nos biênios entre 1997/8 e 2015/6, e foram analisados por Rifai et al. (2018), que constataram que as secas prejudicaram o incremento em diâmetro das árvores, decorrentes da interação entre o déficit da água no solo, a pressão de vapor d'água e a radiação de ondas curtas.

Grimm et al. (1998) defendem que na região sul brasileira, a precipitação é altamente influenciada pelo $E I$ Niño, mas os períodos de estiagens entre junho e dezembro são justificados principalmente pela La Niña, quando ocorrem anomalias climáticas continentais devido a um efeito contrário, ou seja, ao resfriamento das águas superficiais do Oceano Pacífico. Para o Paraná, Ferreira et al. (2017) mencionaram que os efeitos do El Niño de 2015/6 foram mais rigorosos que os verificados em 1982/3 e em 1997/8, e no geral, indicaram aumento de temperatura e de chuvas no estado.

As alterações climáticas aqui reportadas foram analisadas para os meses de inverno, onde no Sul do Brasil, as chuvas são provocadas principalmente pela ocorrência de sistemas frontais, ocasionados pela interação entre a massa de ar polar atlântica com as massas de ar tropical continental e tropical atlântica, mais atuante nas estações de verão e outono, decorrentes de eventos de ENOS. Em síntese, uma provável justificativa para a severa estiagem no inverno de 2015 na área de estudo, face ao aumento de chuvas causadas no Paraná nestes anos, seria que nesta época, massas de ar quente estacionárias de El Niño realizaram bloqueios atmosféricos, impedindo assim o avanço de frentes frias, diminuindo drasticamente as chuvas naquela localidade. Isto porque no biênio anterior (2013/4) e no ano seguinte de 2016 , as chuvas foram significativamente superiores à toda a série analisada, e provavelmente por conta da interação entre massas de ar de El Niño ainda não impeditivas, que em integração às massas polares, teriam favorecido ao aumento das chuvas.

\section{Declínio das árvores maduras}

Pergunta-se se a temperatura elevada e a secura do ar em momentos pontuais poderiam estar favorecendo processos de desvitalização nos pinheiros. Martins-StPaul (2017) e Flexas et al. (2018) demonstraram que o estresse em árvores será maior tanto quanto menor for a capacidade da planta em regular o fechamento dos estômatos foliares, no processo de perda hídrica pela condutância fisiológica, gerando falhas físicas sobre o sistema cambial. Da histologia vegetal, conceitua-se que o felogênio ou "câmbio da casca", forma tanto células do súber (casca externa), quanto internas (feloderme). Para os pinheiros, existe outra camada de câmbio, mais interna ao tronco, responsável pela formação de células internas do xilema, e externas do floema. Ao se considerar que o lenho juvenil e tardio em plantas maduras já estaria com o crescimento estabilizado, este estresse poderia ser verificado em qualquer estação do ano.

Sobretudo, danos físicos poderiam ser verificados pelo efeito da demanda por água destas árvores nos períodos secos, realizadas a partir de chuvas, que caso ocorram intensivas e mal distribuídas, poderiam danificá-las. Acredita-se que as araucárias antigas podem sofrer estresse fisiológico em processos que podem evoluir para a murcha e ressecamento das raízes e dos troncos, causando perda progressiva de biomassa verde nas copas.

O fenômeno das "murchas por ressecamento" foi descrito por Boyce (1948), sendo associado a processos de estresse nas raízes, por impossibilidade de realizarem as trocas gasosas. Anderegg et al. (2016) também descreveram o processo, conceituando-o como "embolia" ou obstrução dos vasos, que ocorre devido à formação de bolhas de ar dentro no sistema hidráulico das árvores, causando tensões mecânicas no transporte da seiva, no momento em que existam tanto baixa umidade no solo quanto elevada demanda de evapotranspiração pelas árvores.

Hartmann et al. (2018b) argumentam que as árvores podem morrer pelos seguintes motivos: i) falhas hidráulicas (hydraulic failure); ii) "fome" de carbono (carbon starvation) ou aqui denominada como Demanda (fotossintética) Não Realizada (DNR); iii) ataques de agentes bióticos; iv) pela interação entre estes fatores. Os autores ainda destacam que conhecimentos sobre 
ecofisiologia vegetal, como o cessamento da respiração noturna e a mortalidade das células do câmbio, podem ser utilizados como sinais de diagnóstico de estresse por causas abióticas, justificando a necessidade de conhecer o point of no return, ou seja, o momento em que as plantas não consigam mais se recuperar.

Gessler et al. (2018) analisaram as relações entre a incapacidade hidráulica e a demanda fotossintética de plantas, tendo proposto uma estrutura conceitual para a compreensão dos efeitos das secas sobre o seu estresse ecofisiológico. Para os autores o declínio das árvores com crescimento estagnado tende a estar associado principalmente à demanda fotossintética não realizada. Em outras palavras, a demanda fotossintética das árvores maduras, associada ao uso ineficiente da água disponível, influenciará a mortalidade do vegetal, que nestes casos ocorrerá lentamente, em função dos fatores ambientais.

Cailleret et al. (2017) ratificaram que a morte das espécies arbóreas em diferentes regiões do planeta é sempre precedida de uma fase de decréscimo ou estagnação do desenvolvimento vegetal. Para Adams et al. (2017), as falhas hidráulicas no xilema são o fator mais significativo para causar a mortalidade de diversas espécies arbóreas, sendo que a partir da perda de $60 \%$ ou mais da condutividade hídrica, o processo de declínio já se torna irreversível.

Aragão et al. (2018) verificaram o aumento na ocorrência de incêndios florestais na Amazônia decorrentes da seca de 2015. McDowell (2018) reitera que períodos de secas são sempre sucedidos de aumento de mortalidade de plantas, e existe forte probabilidade de aumento do declínio das florestas tropicais, devido à diminuição dos intervalos entre as secas provocadas pelo El Niño. O autor também afirma que as espécies arbóreas tenderão a alocar suas energias para investirem em estratégias de defesa e desenvolvimento de estruturas radiculares, ou para reparo hidráulico, mas o equilíbrio trófico dos ecossistemas estará ameaçado por agentes bióticos como as lianas, que se desenvolvem melhor nestes períodos secos e prejudicam as árvores.

Moraes et al. (2018) acreditam que as mudanças climáticas podem estar favorecendo o aumento das chuvas no Rio de Janeiro. Carvalho (2018) analisou o clima nas regiões paranaenses e paulistanas, tendo afirmado que o El Niño de 2008 pode ter influenciado o padrão pluviométrico em 2009. Estas menções indicam que para a área aqui analisada, as árvores maduras podem ter sido também influenciadas pelo El Niño, tendo ficado estressadas nas secas e aumentado sua demanda fotossintética e hidráulica.

\section{CONCLUSÕES}

As mudanças climáticas causadas pelo El Niño são cíclicas e os padrões puderam ser verificados na região de Palmas, General Carneiro e Bituruna no Paraná. Os dados apresentados neste artigo indicam, sobretudo, que os invernos de 2009 e de 2015 foram estressantes às araucárias maduras, dada à irregularidade na distribuição das chuvas e aos períodos de secas. Ainda, que uma próxima estiagem prolongada poderá ser verificada até o inverno do ano de 2021.

O que se hipotetiza é que as anomalias climáticas por ENOS estarão no longo prazo, influenciando esta região com invernos menos frios e com pancadas de chuvas mais intensas. A depender do comportamento da quantidade e distribuição das chuvas, novos ciclos de estresse poderão ser observados, indicando o provável comprometimento da sanidade e da saúde das árvores com baixa resiliência ambiental, com expectativa de aumento de risco de mortalidade destes indivíduos.

Agrega-se que, para o caso das matas com araucárias, certamente, localizadas muito distantes de centros industriais, ou mesmo distante das queimadas na Amazônia, onde a emissão de gases de efeito estufa seja mais preocupante, é que estes declínios e distúrbios florestais estejam também relacionados à falta de intervenções de manejo na floresta, que favoreçam a regeneração natural de árvores do futuro, que contribuam com o bem estar ecológico e a conservação da biodiversidade, favorecendo o controle natural de agentes biológicos infestantes, a fixação de carbono e a produção de serviços ecossistêmicos para a humanidade.

\section{AGRADECIMENTOS}

Ao Engenheiro Florestal Luciano Pizzatto (in memorian) e às Indústrias Pedro Pizzatto Ltda., por permitirem a coleta de dados para as análises fitossanitárias, e por não desistirem de fomentar o debate acerca do manejo dos recursos da mata atlântica.

\section{REFERÊNCIAS}

ADAMS, H.D. et al. A multi-species synthesis of physiological mechanisms in drought-induced tree mortality. Nature ecology \& evolution, v.1, n.9, p.1285-1291, 2017.

ALVARES, C.A. et al. Köppen's climate classification map for Brazil. Meteorologische Zeitschrift, v.22, n.6, p.711-728, 2013.

ANDEREGG, W.R.L. et al. Meta-analysis reveals that hydraulic traits explain cross-species patterns of drought-induced tree 
mortality across the globe. Proceedings of the National Academy of Sciences (PNAS), v.113, n.18, p.5024-5029, 2016.

ARAGÃO, L.E.O.C. et al. 21st century drought-related fires counteract the decline of Amazon deforestation carbon emissions. Nature communications, v.9, n.536, p.1-12, 2018.

AUBIN, I. et al. Tree vulnerability to climate change: improving exposure-based assessments using traits as indicators of sensitivity. Ecosphere, v.9, n.2, p.1-24, 2018.

BARTH-FILHO, N. Monitoramento do crescimento e da produção em Floresta Ombrófila Mista com uso de parcelas permanentes. 2002. 112p. (Dissertação de mestrado).

BELL, D.M. et al. Visual interpretation and time series modeling of Landsat imagery highlight drought's role in forest canopy declines. Ecosphere, v.9, n.6, p.1-17, 2018.

BOYCE, J.S. Forest Pathology. 2.ed. Nova lorque: McGraw-Hill, 1948.

BRANDO, P.M. et al. Abrupt increases in Amazonian tree mortality due to drought-fire interactions. Proceedings of the National Academy of Sciences of the United States of America, v.111, n.17, p.6347-6352, 2014.

BRASIL. Decreto federal no. 2.519, de 16 de março de 1998. Diário Oficial da União. Brasília, 1998. Disponível em: http://www.planalto.gov.br/ccivil_03/decreto/D2519.htm

BRASIL. Portaria MMA nº 43 de 31 de janeiro de 2014. Diário Oficial da União. Brasília, 2014.

BRASIL. Portaria MMA nº 443 de 17 de dezembro de 2014. Diário Oficial da União. Brasília, 2014.

CAILLERET, M. et al. A synthesis of radial growth patterns preceding tree mortality. Global Change Biology, v.23, n.1, p.1675-1696, 2017.

CARVALHO, S.M.I. Influencia da variabilidade climática na dinâmica da vegetação na região da Mata Atlântica Paulista. 2018. 137p.

CASTRO-FARIA, A.B. Conservação e Saúde das Araucárias: fundamentos legais e ecossistêmicos. Curitiba: Juruá, 2018.

DALE, V.H. et al. Climate Change and Forest Disturbances: Climate change can affect forests by altering the frequency, intensity, duration, and timing of fire, drought, introduced species, insect and pathogen outbreaks, hurricanes, windstorms, ice storms, or landslides. Bioscience, v.51, n.9, p.723-734, 2001.

DALLA-CORTE, A.P. et al. Dinâmica do crescimento, mortalidade e recrutamento na floresta com araucária. Floresta com Araucaria: Pesquisas Ecológicas de Longa Duração. Curitiba: Multigraphics, 2014.

FANG, O. et al. Tree rings reveal a major episode of Forest mortality in the late 18th century on the Tibetan Plateau. Global and Planetary Change, v.163, p.44-50, 2018.
FERREIRA, L.G.B. et al. O fenômeno EI Niño de 2015/2016 e seus impactos nas chuvas do Paraná. Londrina: IAPAR, 2017.

FLEXAS, J. et al. Gas Exchange and hydraulic during drought in crops: who drives whom? Journal of Experimental Botany, v.69, n.16, p.3791-3795, 2018.

FRITZSONS, E. et al. Análise da pluviometria para definição de zonas homogêneas no Estado do Paraná. RAEGA, v.23, n.1, p.555-572, 2011.

FRITZSONS, E.; WREGE, M.S. A distribuição natural do Pinheirodo-Paraná no Sul e Sudeste do Brasil: a influência de fatores climáticos. Colombo: Embrapa, 2017.

FRITZSONS, E. et al. Fatores climáticos limitantes da distribuição da araucária no estado do Paraná e as implicações para sua restauração. RAEGA, v.44, n.1, p.258-271, 2018.

GENTILESCA, T. et al. Drought-induced oak decline in the western Mediterranean region: an overview on current evidences, mechanisms and management options to improve forest resilience. iforest, v.10, n.5, p.796-806, 2017.

GESSLER, A. et al. Drought induced tree mortality - a tree-ring isotope based conceptual model to assess mechanisms and prepositions. New Phytologist, v.218, n.2, p.485-490, 2018.

GRIMM, A.M. et al. Precipitation anomalies in Southern Brazil associated with El Niño and La Niña events. Journal of climate, v.11, n.1, p.2863-2880, 1998.

HARTMANN, H. et al. Research frontiers in drought-induced tree mortality: crossing scales and disciplines. New Phytologist, v.205, n.1, p.965-969, 2015.

HARTMANN, $\mathrm{H}$. et al. Monitoring global tree mortality patterns and trends. Report from the VW symposium "Crossing scales and disciplines to identify global trends of tree mortality as indicators of forest health". New Phytologist, v.217, n.1, p.984987, 2018a.

HARTMANN, $\mathrm{H}$. et al. Research frontiers for improving our understanding of drought-induced tree and forest mortality. New Phytologist, v.218, n.1, p.15-28, 2018b.

INTERGOVERNMENTAL PANEL ON CLIMATE CHANGE (IPCC). Climate Change 2014: Synthesis Report - Contribution of Working Groups I, II and III to the Fifth Assessment Report of the IPCC. Genova: IPCC, 2014.

JOHNSON, R.A.; WICHERN, D.W. Applied multivariate statistical analysis. 6.ed. New Jersey: Prentice-Hall, 2007.

MAIA, A.G. et al. Climate change and agriculture: do environmental preservation and ecosystem services matter? Ecological Economics, v.152, p.27-39, 2018.

MALTHI, Y. et al. New insights into the variability of the tropical land carbon cycle from the El Niño of 2015/2016. Philosophical Transactions of the Royal Society B: Biological Sciences, v.373, p.1-7, 2018. 
MANION, P.D. Tree diseases concepts. 2.ed. Chicago: Prentice Hall, 1991.

MARTINS-STPAUL, N. et al. Plant resistance to drought depends on timely stomatal closure. Ecology letters, v.20, n.11, p.1-11, 2017.

McDOWELL, N.G. Deriving pattern from complexity in the process underlying tropical forest drought impacts. New Phytologist, v.219, n.3, p.841-844, 2018.

MEZA, F.J. et al. Soil water content effects on net ecosystem $\mathrm{CO}_{2}$ exchange and actual evapotranspiration in a Mediterranean semiarid savanna of Central Chile. Nature scientific reports, v.8, n.8570, p.1-11, 2018.

MOGNON, F. et al. Uma década de dinâmica da fixação de Carbono na Biomassa arbórea em Floresta Ombrófila Mista no Sul do Paraná. Floresta, v.43, n.1, p.153-164, 2013.

MORAES, J.O.C. et al. The influence of climate change on the intensity and distribution of the rain in four Rio de Janeiro weather stations. Ambiência, v.14, n.1, p.140-153, 2018.

NELSON, H.P. et al. A call to action for climate change research on Caribbean dry forests. Regional Environmental Change, v.18, n.5, p.1337-1342, 2018.

PÉREZ, C.A.; ARNESTO, J.J. Coupling of microbial nitrogen transformations and climate in sclerophyll forest soils from the Mediterranean Region of central Chile. Science of the Total Environment, v.625, p.394-402, 2017.

RIBAS, J.H.R.; VIEIRA, P.R.C. Análise Multivariada com o uso do SPSS. Rio de Janeiro: Ciência Moderna, 2011.

RIFAI, S.A. et al. ENSO drives interannual variation of forest woody growth across the tropics. Philosophical Transactions of the Royal Society B, v.373, n.1760, p.1-13, 2018.

SALVIANO, M.F. et al. Análise de tendências em dados de precipitação e temperatura no Brasil. Revista Brasileira de Meteorologia, v.31, n.1, p.64-73, 2016.

SAS INSTITUTE. The GLIMMIX Produce. SAS/STAT 9.2 User's Guide. Cary: SAS Institute, 2008.

SAS INSTITUTE. Base SAS 9.4 Procedures Guide: Statistical Procedures. 2.ed. Cary: SAS Institute, 2013.

SHENKIN, A. et al. Interactive effects of tree size, crown exposure and logging on drought-induced mortality. Philosophical Transactions of the Royal Society B: Biological Sciences, v.373, p.1-10, 2018.

SUGDEN, A. et al. Forest health in a changing world. Science, v.349, n.6250, p.800-801, 2015.

TRUMBORE, S. et al. Forest health and global change. Science, v.349, n.6250, p.814-818, 2015.

WESTERLING, A.L. et al. Warming and earlier spring increase Western U.S. forest wildfire activity. Science, v.313, n.5789, p.940-943, 2006.
WREGE, M.S. et al. Principais fitofisionomias existentes no estado do Paraná e os novos cenários definidos pelas mudanças climáticas globais. Ambiencia, v.13, n.3, p.600-615, 2017. 